\title{
New technique of stereolithography to local curing in thermosensitive resins using $\mathrm{CO}_{2}$ laser
}

\author{
A.L.J. Munhoz ${ }^{(*)}$, P. R. S. Wagner $\left(^{(*)}, \mathrm{M}^{\text {a }}\right.$ C. F. Ierardi $\left(^{(*)}\right.$, A.E. Kiel ${ }^{(* *)}$, M.A F. \\ Scarparo $^{(* *)}$ y S.D.Allen ${ }^{(* * *)}$
}

Abstract: A theoretical and experimental study of thermosensitive resins used in thermal stereolithography is presented. The process of local curing through the application of infrared radiation, which has proved to be useful in a new technique for the making of prototypes by means of selective heating with $\mathrm{CO}_{2}$ laser $(10.6 \mu \mathrm{m})$, is studied. The ideal composition of the thermosensitive resins has proved to be 10 parts epoxy, 1.4 part diethylene triamine (the curing agent) and 0.7 part silica powder. A physical theoretical model is applied for control of the parameters which influence the confinement of the curing in the irradiated bulk. A mathematical model is applied too; it was developed through the resolution of the heat conduction equation dependent on time in cylindrical co-ordinates, which enables to determine the behaviour of curing in terms of irradiation conditions.

Keywords: Laser. $\mathrm{CO}_{2}$. Thermosensitive resins. Stereolithography.

\section{Nueva técnica de estereolitografía para cura localizada en resinas termosensibles usando el láser de $\mathrm{CO}_{2}$}

Resumen: Se presenta un estudio teórico y experimental, sobre resinas termosensibles usadas en estereolitografia térmica. Se estudia el proceso de cura local mediante la aplicación de radiación infrarroja producida por el láser de $\mathrm{CO}_{2}(10.6 \mu \mathrm{m})$, el cual mostró ser útil en técnicas nuevas, para la fabricación de prototipos por medio de calentamiento selectivo. La composición ideal para cura local de las resinas termosensibles, está en la proporción de 10.0 partes de epoxi, 1.4 partes de dietilentriamina y 0.7 partes de sílice. Para controlar los parámetros que influyen en el confinamiento de la cura a través de la irradiación, se utiliza un modelo teórico-físico. Se utiliza un modelo matemático en coordenadas cilíndricas basado en la ecuación de conducción de calor dependiente del tiempo, que permite determinar el comportamiento de cura en función de las condiciones de irradiación.

Palabras clave: Láser. $\mathrm{CO}_{2}$. Resinas termosensibles. Estereolitografía.

\section{INTRODUCTION}

The great world movements in search of optimization in prototype production has placed

(*) State University of Campinas - Unicamp, Mechanical Engineering Faculty (DEMA). C.P 6122 - 13083-970 Campinas - SP - (Brazil). amunhoz@fem.unicamp.br.

(**) State University of Campinas - Unicamp, Institute of Physics (DEQ). Lab. of Laser Application to Fine Mechanics. C.P 6165 - 13083-970 - Campinas - SP (Brazil).scarparo@ifi.unicamp.br.

(***) Florida State University - 217, Westcott Building Tallahassee (FL, USA) - 32306-4093. stereolithography as a powerful technique used for obtaining tridimensional models. Usually, the conventional technique in stereolithography makes use of systems of application of ultraviolet light on photosensitive resins in the curing process, by operating a $\mathrm{HeCd}(0.352 \mu \mathrm{m})$ laser for obtaining prototypes $(1 \mathrm{y} 2)$. This work presents the study of an innovator technique of application of $\mathrm{CO}_{2}(10.6$ $\mu \mathrm{m})$ laser infrared radiation on thermosensitive resins by means of stereolithography (3). It is believed to be a really new and advantageous method as compared with the process mentioned before. In previous works, the behaviour of the influence parameters as well as the characteristics 
of the resins in the process of local curing were studied and defined (4). The results were satisfactory as a starting point for the determination of a proper theoretical model (5), aiming at the accurate determination of the local curing. In order we might come to such a composition for local curing, a detailed study was fulfilled considering the amount of silica in the process. The experimental analysis were carried out in two stages -one of thermal characterisation and another of optical characterisation of the thermosensitive resins. The results determined the temperature range at which the curing starts and the behaviour of the absorption depth in terms of the silica variation in the composition. A mathematical model was applied by solving the equation for the conduction of heat dependent on time in the cylindrical co-ordinates, which permits to foresee the behaviour of the curing in terms of irradiation conditions.

\section{THEORETICAL MODEL}

A physical theoretical model (6) has been worked out aiming at the exact characterisation of every physical phenomenon occurred in the process of local curing. The model describes the energy flow deposited by the laser in terms of the control of the operational parameters and the behaviour of the resin, aiming at the local curing. The determination of how the released energy was distributed is essential for obtaining the local curing. The local curing was achieved by scanning a continuous wave (cw) $\mathrm{CO}_{2}$ laser repeatedly over a circular trajectory on the sample's surface with a scan speed. By dividing the beam diameter by the scan speed, the 'dwell time':

$$
\tau_{\mathrm{d}}=2 \omega / \mathrm{v}
$$

is obtained concerning the time of interaction laser/resin at a surface point. As the resin is highly absorptive at the $\mathrm{CO}_{2}$ laser wavelength $(10.6 \mu \mathrm{m})$, it is assumed that, during the 'dwell time', nearly all the beam energy has gone into the inner part of the sample at a distance from the surface equivalent to the absorption depth, $\delta$. It is assumed that energy $E$ has been absorbed in the small cylindrical volume $V$ during the 'dwell time', the volume being defined as:

$$
V=\pi \omega^{2} \delta
$$

The energy released in $V$ is the product of the laser power by 'dwell time':

$$
E=P \tau_{\mathrm{d}}
$$

By means energy, $E$, it is possible to determine the variation of temperature, which is proportional to the deposited energy concerning to specific heat, $\mathrm{C}_{\mathrm{p}}$, and mass, $m$, of the material contained in volume, $V$, according to the following equation:

$$
E_{\mathrm{p}}=C_{\mathrm{p}} \mathrm{m} \Delta T
$$

The mass of the heated volume may be calculated by using the mass density of the sample $\rho=1,16$ $\mathrm{g} / \mathrm{cm}^{3}$. A numerical solution was applied based on the Finite Differences Method, developed to the general equation for conduction of heat (7). If it is assumed that nearly all the flow of energy deposited by the laser beam is absorbed every moment the laser passes at a point on the surface of the sample, it follows that the irradiated volume will undergo a temperature increase which is determined by the expression:

$$
\Delta T=\frac{E_{\mathrm{p}}}{m C_{\mathrm{p}}}=\frac{P \tau_{\mathrm{d}}}{\pi \omega^{2} \delta \rho C_{\mathrm{p}}}
$$

As it concerns to a laser linear heating rate, the general equation for conduction of heat dependent on time is applied as follows:

$$
\nabla^{2} T=\frac{1}{D} \frac{\partial T}{\partial T}-\frac{G}{K}
$$

where $D$ stands for the thermal diffusivity of the sample, $K$ is the thermal conductivity, and $G$ describes the rate of energy generated by the laser source. As it concerns to a Gaussian profile (8) of intensity of the laser beam, the term of source $G$ generated by the $\mathrm{CO}_{2}$ laser may be expressed by:

$$
G=\frac{P}{\pi \omega^{2} \delta} \exp ^{\left(-\mathrm{r}^{2} / \omega^{2}\right)} \exp ^{\left(\frac{-z}{\delta}\right)} \mathrm{s}\left(\tau_{\mathrm{d}}\right)
$$

for $0 \leq \mathrm{t} \leq \tau_{\mathrm{d}}, \mathrm{S}\left(\tau_{\mathrm{d}}\right)=1 ; \mathrm{t}>\tau_{\mathrm{d}}, \mathrm{S}\left(\tau_{\mathrm{d}}\right)=0$ where $\mathrm{S}\left(\tau_{\mathrm{d}}\right)$ is the function which considers the term of source in the general equation, $r$ is the distance from the beam center, $z$ is the depth of the sample surface, $\delta$ is the absorption depth, $P$ is the output power of the laser.

The displacement scan speed over the trajectory of the circular way resulted in repetition rate of the laser equal to , and the 'dwell time' of $377 \mu$ s for a beam focused to of the radius. These parameters were used vin the numerical simulation, the constant of radial thermal transient $\sigma$ being defined as follows: $=\omega^{2} / \mathrm{D}$, where $\mathrm{D}=22 \times 10^{-5} \mathrm{~cm}^{-2} \cdot \mathrm{s}^{-1}$, thus $\sigma$ is approximately $10 \mathrm{~s}$ for the radial transient. The Crank-Nickelson method of infinite differences was used to solve the equation for the conduction heat 
dependent on time in the cylindrical co-ordinates. The thermal conductivity of the epoxy sample is $\mathrm{K}=0.359 \times 10^{-3} \mathrm{~mW} / \mathrm{cmK}$, which is near the conductivity of air $\mathrm{K}=0.24 \times 10^{-3} \mathrm{~mW} / \mathrm{cmK}$. As it concerns to the theoretical model, it was assumed that thermal properties $K$ and $D$, as well as the optical properties $\delta$ and reflectivity are all of them independent of temperature. The influence of silica in the process of local curing was disregarded.

\subsection{Experimental Analysis}

\subsubsection{Stage I}

Initially, for the thermal characterization of the thermosensitive resin, it was essential the study of the sample behaviour under the influence of the heat action. For that purpose, it was decided to simulate experimentally how the sample reacts when it is submitted to an external source of heat. In order to carry out the experimental analysis, the experimental apparatus was used, in which a small semi-liquid volume of the sample was heated at different external temperatures. Although the result of the local curing may also be obtained with polyester resin, the decision to use the epoxy resin (\# D.E.R. 330) was taken because of its low coefficient of thermal diffusivity $\mathrm{D}=22 \times 10^{-5} \mathrm{~cm}^{2} \cdot \mathrm{s}^{-1}$, as well as its appropriate viscosity, thermosensitivy and stability in the course of the curing process. In order that the explanations might be coherent, we were firstly decided to characterise the curing process occuring in the bulk of the sample. For that purpose, a small volume of liquid sample was heated in a beaker wrapped in heat tape, which enabled to control the parameters which could bring information concerning the curing process. Initially, the temperature of the sample volume in terms of time was monitored by means of a thermocouple by applying different surrounding temperatures (external). Temperatures of 37, 44, 57, 65, 73 and 80 ${ }^{\circ} \mathrm{C}$ were selected for the analysis of the curing behaviour of the sample. The results obtained in the graphics initially indicate a similar behaviour in the evolution process of the curing at the different temperatures applied; however, it is clearly observed a much faster answer to the curing as the temperature increases. It is also noticed that the onset of the curing process occurs in a very narrow temperature range of the sample (onset the curing). Such a variation arises out of the difficulty in controlling stoichiometry when preparing the sample.

\subsubsection{Stage II}

The optical characterization of the properties of the thermosensitive resin will depend on the sample composition, which in proper conditions permits obtaining information about the absorption depth. Therefore, these analyses have proved to be of great use for the study of the samples behaviour in terms of the silica absorption of energy, since each sample was submitted to a variation in the amount of silica in the composition. The absorption depth, as defined in the theoretical physical model, is essential for the definition of the volume of the cured sample. If the absorption depth determines the radiation depth in the sample, it follows that the amount of silica in the composition may determine the dimension of the cured volume, seeing that as the amount of silica changes, there occurs a variation in the absorption depth. In order to determine the absorption depth in each sample, since the semi-liquid sample was inserted between two $\mathrm{KBr}$ crystals (transparent to infrared light). An analytical solution -by applying the Lambert-Beer Law $\left(I_{\mathrm{T}}=I_{0} e^{-\mathrm{x} / \delta}\right)$ was established. Considering that the intensity is partly reflected and attenuated by the crystals; through the analytical solution it is possible to determine the depth of energy absorption by the sample.

Thus, by applying the Lambert-Beer Law, a satisfactory estimate is obtained as follows:

$$
I_{\mathrm{T}}=I_{0}(1-R)^{2} e^{-\alpha_{\mathrm{c}}\left(\mathrm{x}_{\mathrm{cl}}+\mathrm{x}_{\mathrm{c} 2}\right)} \mathrm{e}^{-\frac{\mathrm{x}_{\mathrm{a}}}{\delta_{\mathrm{a}}}}
$$

where: $I_{\mathrm{T}} / I_{0}=$ sample transmittance, $R=\mathrm{KBr}$ crystal reflectance, $x_{\mathrm{c}}=$ crystal thickness, $\alpha=$ optical absorption coefficient of the crystal, $\delta=$ absorption depth.

If $\delta$ is detached from eq, [8], it follows:

$$
\delta_{\mathrm{a}}=-\frac{\mathrm{x}_{\mathrm{a}}}{\alpha_{\mathrm{c}}\left(\mathrm{x}_{\mathrm{cl}}+\mathrm{x}_{\mathrm{c} 2}\right)+\ln \left[T \frac{1}{(1+R)^{2}}\right]}
$$

where $T$ stands for transmittance. The analysis of the optical absorption depth of the samples have shown how silica is important in the calculation of the samples behaviour under the influence of infrared radiation. In order to facilitate the accomplishment of these experiments, the proportion of the silica in the composition (which permits the local curing of the resin) was considered as the basis, where for each sample composition the following proportions were added in parts of the reagents.

\section{RESULTS AND DISCUSSIONS}

The experiments concerning samples under exothermic conditions indicate the possibility of influencing the curing of the sample through the 
control of factors which affect the speeds of reaction, and determine the moment the reaction starts. As suggested by the experiment in question it was important because, traditionally, it is possible to determine the activation energy of the reaction involved in the curing process with the obtaining of the complete temperature curve for the sample in the function of time. This result permits to establish a mathematical relationship between the parameters involved in the curing and to obtain the activation energy of the reaction.

However, the experimental difficulties are clear along this process, which does not make it possible to obtain the activation energy accurately, since the highest temperature point of reaction has not been attained. For example, to determine the reaction rate more accurately, as a function of temperature, DSC (differential scanning calorimetry) should be used. The study of the sample by means of DSC is being developed and aims to determine the conversion rate of the sample in the curing, in function of time as well as to determine the activation energy of the sample, which is estimated to be around $\mathrm{E}_{\mathrm{a}}=50 \mathrm{kcal} / \mathrm{mol}$. The simple analytical solution established in terms of the sample transmittance and thickness was used for the analysis of the behaviour of the absorption depth in semi-liquid samples with variation of silica in the composition.

From a theoretical and experimental viewpoint, silica plays an important part in controlling the local curing, as it hinders heat diffusion to zones which are outside of the irradiated area. The amount of silica is understood to be critical in the process of local curing, since if silica occurs

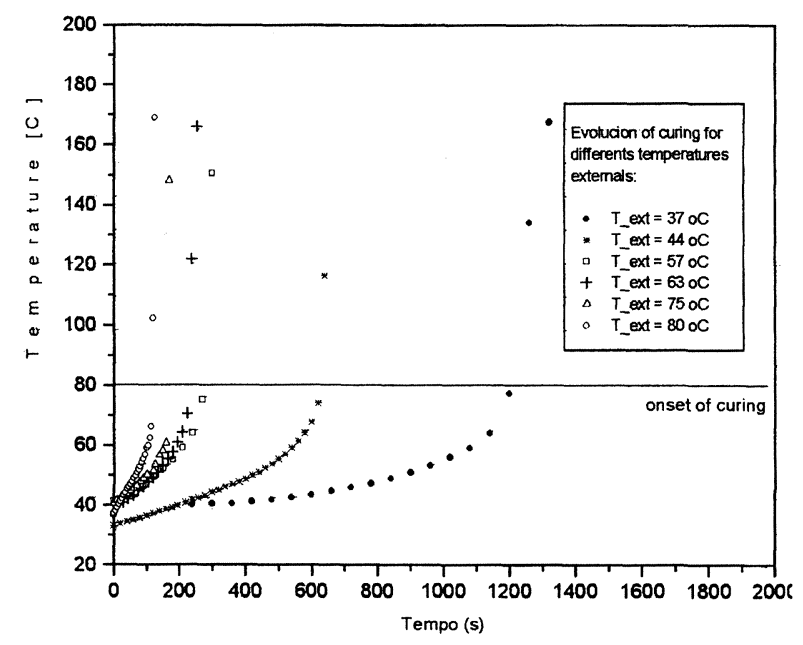

FIG. 1.- Evolution of the sample internal temperature and onset of curing for different external temperatures.

FIG. 1.- Equipamiento experimental en la caracterización del proceso de cura. excessively in the composition, it restricts the curing of the reagents and absorbs all the energy. Thus, the curing is not complete, even if it occurs. On the other hand, if silica occurs in a small amount, heat may diffuse to undesirable areas, which facilitates the curing among the reagents and makes the local curing impracticable. Notwithstanding, it is possible to control the thickness of the cured layers when the absorption depth is known through the control of the silica amount added to the composition. Thus, the energy penetration in the sample is defined. The interest in analyzing in terms of the variation of silica powder is associated with the obtaining of local curing, since it is the amount of silica powder that will determine the depth of energy entering into the irradiated sample (Fig. 2), and consequently the thickness of the cured part. Considering the importance of silica in the formation of the appropriate composition of the thermosensitive resin, it is indispensable to analyse the effect since it is considered to be one of the main parameters in the physical interpretation and development of the process to obtain local curing.

A further preoccupation was to create a physical model with laser operational parameters which could determine the boundary conditions of the laser application on the sample for the obtaining of the local curing. A careful control of these parameters (scan speed, power output and depth absorption) is essential for the confinement of the curing in the volume specified in the eq.[2]. In order to know the importance of the parameters which are involved, an experiment was made by scanning the beam through a circular trajectory at

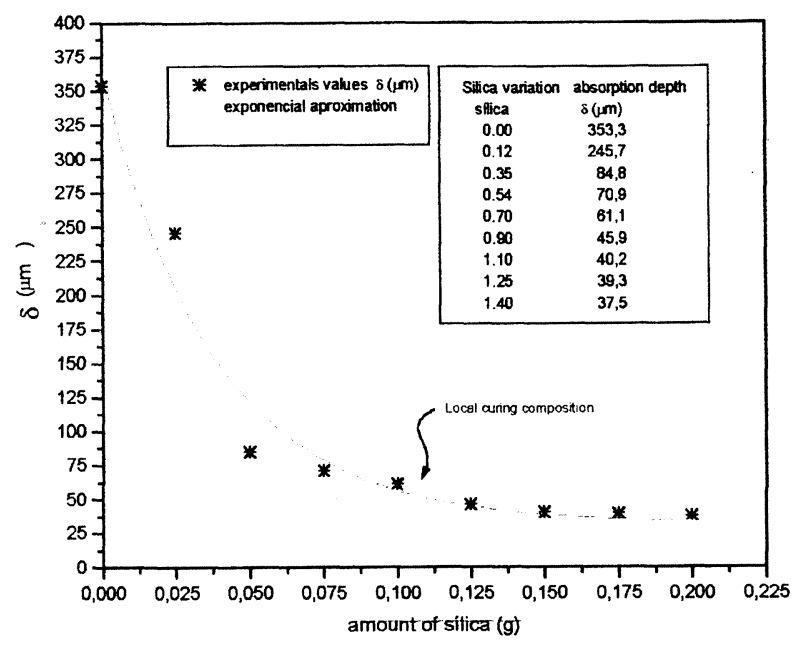

FIG. 2.- Depth of optical absorption in terms of silica content variation.

FIG. 2.- Profundidad de absorción óptica en función de la variación del contenido de sílice. 


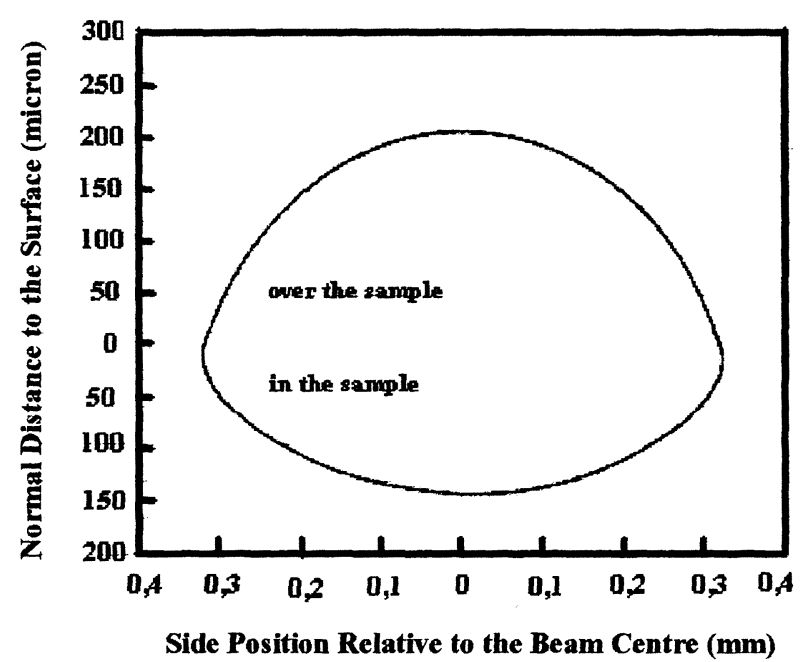

FIG. 3.- Isothermal mapping for $1 / e$ of the highest temperature in the sample volume.

FIG. 3.- Mapeamiento isotérmico para l/e de la temperatura máxima en el volumen de la muestra.

the sample surface, at a scan speed $v=159.2$ $\mathrm{cm} \cdot \mathrm{s}^{-1}$, which results in a laser repetition rate of 35 ms, a dwell time of $\tau_{\mathrm{d}}=377 \mu$ s was obtained. The laser beam was focused to $1 / e$ of the $0.3 \mathrm{~mm}$ radius, so that by using an absorption depth of $\delta=$ $22.4 \mu \mathrm{s}$, the energy deposited by the laser at each 'dwell time' in the $0.33 \times 10^{-2} \mathrm{~mm}^{-3}$ volume was calculated. The laser being operating continuously with $\mathrm{P}=20 \mathrm{~W}$, the deposited energy was found to be $\mathrm{E}_{\mathrm{p}}=7.6 \mathrm{~mJ}$ approximately, the numerical model has shown to be quite flexible on describing the complete bidimensional mapping of the thermal evolution in the sample bulk. The distribution of temperature at each point of the attained volume and over the sample surface was obtained through numerical simulation. Figure 3 shows the distribution of temperature along areas situated over and below the sample surface with the isothermal profile described for $1 / e$ of the highest temperature reached in the sample. The results produced by the numerical model proved the possibility of confining the curing within the same dimension of the laser beam. They are in conformity with the preliminary experimental results where tridimensional pieces were built by means of the superposition of layers with individual thickness varying from 0.1 to $0.2 \mathrm{~mm}$.

\section{CONCLUSIONS}

The results of the study in the process of local curing of a semi-liquid sample, composed of epoxy resin, diethylentriamine and powdered silica, with the use of a $\mathrm{CO}_{2}$ laser as selective source of heat, were presented. The use of infrared laser to locate the curing of thermosensitive resins is new, and it is believed to be an advantageous method as compared with the conventionally used process. It was observed that local curing only takes place with a specific composition of the sample. In order that the curing might occur, the sample surface was swept by the laser beam along a circular trajectory, at an appropriate speed. In order to adapt the process of local curing, with the use of infrared radiation, for the obtaining of plastic pieces of unlimited geometry, it is necessary the effective control of the parameters involved, including laser power, 'dwell time' or exposition time, speed and frequency of displacement, the laser beam dimension, the thermal conductivity of the sample, and the reagents mixture. Numerical simulation has proved successful as it concerns the lateral confinement and the depth of the curing as dimensioned by the laser beam, with excellent spatial resolution, and with no shrinkage of the sample after the curing, to the detriment of the final product. Interesting results were obtained with the experiment related to the application of external temperature to the sample, which affects the reaction speed of the curing. For this experiments it is concluded that, if the boundary conditions are improved, it will be possible to obtain, for example, the activation energy of the reaction for the sample in question at a low cost, in a simpler experiment, since the reaction is exotermic. The application of DSC is already renowned and involves sophisticated equipment of high cost. However, it presents very good results for the obtaining of the activation energy of the reaction.

\section{REFERENCES}

(1) Kaplan, R. Photonics Spectra. Jun. 1990: 74-75.

(2) Belfore, D.A. Laser Focus World. Jun 1993: 26-131.

(3) Barros, M.L., Scarparo, M.A.F. and Gerck, E. Stereolithography: A New Technique, in V State Symposium of Laser e Applications. IPEN-SP-BRASIL. 1992

(4) Barros, M.L., Scarparo, M.A.F. Fast Prototyping with Thermal Stereolithography. J. Braz. Soc. Mechan. Sci., XVI (1), 1994: 51-57.

(5) Scarparo, M.A.F., Chen, Q.J., Miller, A.S., Li, C.J., Barros, M.L., Leary, Hurtak. J., Jr. y Allen, S.D. Localized Curing of Thermosensitive Polymer using Infrared Radiation. Laser Spectroscopy \& Molecular Dynamics: Laser Techniques for Surfaces Science II. San Diego (CAL, USA) Ref. $n^{\circ}$ 2547-2564, 1995.

(6) SCARPARo,M.A.F. et all. Study of Resin Based Materials using $\mathrm{CO}_{2}$ Laser Stereolithography. J. Sci. Tech Polymers. 38 (9), 1997: 2.175.

(7) Carslaw, H.S. and Jaege, J.C., "Conduction of heat in solids", $2^{\text {nd }}$ ed.,New York, 1959.

(8) SAnders, D.J. Temperature Distributions Produced by Scanning Gaussian Laser Beams. Appl. Optics. 23 (1), 1984: 30-35. 